\title{
Hemorrhagic shock occurring due to a concealed hematoma after insertion of a subclavian venous catheter in a patient undergoing anticoagulation therapy
}

\section{-a case report-}

\author{
Boo-Young Hwang ${ }^{1}$, Eunsoo Kim ${ }^{1}$, Won-Sung Kim ${ }^{1}$, Ah-Reum Cho ${ }^{1}$, Mi-Jung Cho ${ }^{1}$, and \\ Chung-Won Lee ${ }^{2}$
}

${ }^{1}$ Department of Anesthesia and Pain Medicine, Pusan National University School of Medicine, ${ }^{2}$ Department of Cardiovascular and Thoracic Surgery, Pusan National University Hospital, Busan, Korea

A 74-year-old man who had been receiving warfarin for atrial fibrillation, underwent emergency thrombectomy. A central venous catheter (CVC) was inserted via the left subclavian vein, and heparin was administered to prevent preoperative and postoperative thrombotic events. After an uneventful thrombectomy, the patient was transferred to the intensive care unit (ICU). On the second postoperative day, the patient developed syncope and his blood pressure and oxygen saturation decreased. A computed tomography (CT) revealed a huge hematoma under the pectoralis major muscle. The patient was then treated with continuous renal replacement therapy and mechanical ventilation for multiorgan dysfunction syndrome, which developed due to hemorrhagic shock in the ICU. These findings suggest that when a CVC is inserted in patients requiring anticoagulant therapy, the possible risk of excessive bleeding must be carefully considered. Further, choosing a proper insertion site and performing an ultrasound-guided aspiration may be helpful in preventing these complications. (Korean J Anesthesiol 2014; 67: 358-362)

Key Words: Anticoagulants, Central venous catheter, Hematoma, Hemorrhagic shock.

Received: August 30, 2013. Revised: 1st, September 17, 2013; 2nd, October 2, 2013. Accepted: October 4, 2013.

Corresponding author: Eunsoo Kim, M.D., Department of Anesthesia and Pain Medicine, Pusan National University School of Medicine, 179, Gudeok-ro, Seo-gu, Busan 602-739, Korea. Tel: 82-51-240-7392, Fax: 82-51-242-7466, E-mail: eunsookim@pusan.ac.kr

(c) This is an open-access article distributed under the terms of the Creative Commons Attribution Non-Commercial License (http:// creativecommons.org/licenses/by-nc/3.0/), which permits unrestricted non-commercial use, distribution, and reproduction in any medium, provided the original work is properly cited. 
Central venous catheters play an important role in the clinical management of patients undergoing major surgery or those with critical illness. However, it can also lead to mechanical complications, venous thrombosis, and catheter-related infections [1-4]. Hematoma and arterial puncture are common complications that can occur during catheterization. Although usually self-limiting entities, these complications may have serious sequelae if they involve the great vessels or if treatment is delayed. Here, we report a case of hemorrhagic shock occurring due to a huge hematoma under pectoralis major muscle, after insertion of central venous catheter in a patient undergoing anticoagulation therapy.

\section{Case Report}

A 74-year-old man with diabetes mellitus (DM), hypertension (HTN), and atrial fibrillation (AF) presented to the emergency room with sudden onset of left leg pain due to superficial femoral artery (SFA) thrombosis. Soon after arrival, he was scheduled to undergo emergency thrombectomy. He had been receiving metformin (850 $\mathrm{mg} /$ day) for DM, olmesartan $(40 \mathrm{mg}$ / day) for HTN, and warfarin (3 mg/day) and digoxin $(0.25 \mathrm{mg} /$ day) for AF. In the preoperative evaluation, chest radiography showed cardiomegaly, while electrocardiogram showed the presence of atrial fibrillation. Laboratory investigations showed no remarkable findings. Prothrombin time (PT) and international normalized ratio (INR) were within the normal range (PT, $10.5 \mathrm{sec}$; INR, 0.92), even though the patient was on warfarin therapy. A central venous catheter (CVC, 7-Fr double lumen catheter) was inserted via the left subclavian vein using the landmark technique under general anesthesia. In the first attempt,

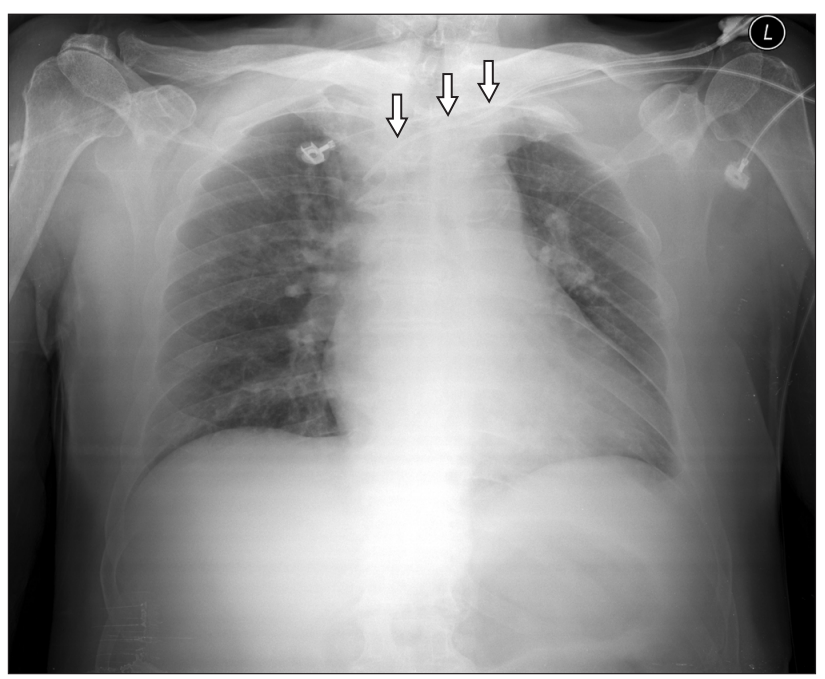

Fig. 1. Chest X-ray after thrombectomy showing the proper position of central venous catheter in the superior vena cava (arrows). the needle was passaged twice without aspiration of blood. In the second attempt, after positioning the needle tip, blood was successfully aspirated through the needle in the third passage to confirm its location, followed by successful insertion of the CVC.

Subsequently, 5,000 U of heparin was administered 5 minutes before incision of the femoral artery for thrombectomy. The thrombectomy was performed uneventfully with an operative time of 90 minutes. The arterial blood gas analysis (ABGA) during surgery showed the following: $\mathrm{pH}, 7.337 ; \mathrm{PaCO}_{2}, 43.4$ mmHg; $\mathrm{PaO}_{2}, 112 \mathrm{mmHg}$; and oxygen saturation, 98\%. Additionally, the hemoglobin $(\mathrm{Hb})$ level was $15.6 \mathrm{~g} / \mathrm{dl}$. The estimated blood loss and urine output were approximately $100 \mathrm{ml}$ and 300 $\mathrm{ml}$, respectively. A total of $1,250 \mathrm{ml}$ of Hartmann's solution was administered during surgery.

The patient was then transferred to intensive care unit (ICU) for close monitoring. Postoperatively, continuous infusion of heparin $(1,000 \mathrm{U} / \mathrm{hr})$ via CVC was maintained. The infusion rate was adjusted under the activated partial thromboplastin time of 80-120 sec. A portable chest radiograph showed proper position of the CVC in the superior vena cava and no interval changes (Fig. 1). At $6 \mathrm{hr}$ postoperation, a small swelling and oozing were observed at the insertion site of the CVC. Consequently, CVC was removed and compressed for 30 minutes. At $15 \mathrm{hr}$ postoperation, when the patient's hemodynamic values were stable and the dorsalis pedis artery was palpable, he was transferred to the general ward. On the first postoperative day (POD), the patient was started on warfarin ( $4 \mathrm{mg} /$ day). A chest radiograph at this point showed no remarkable findings except a slightly increased

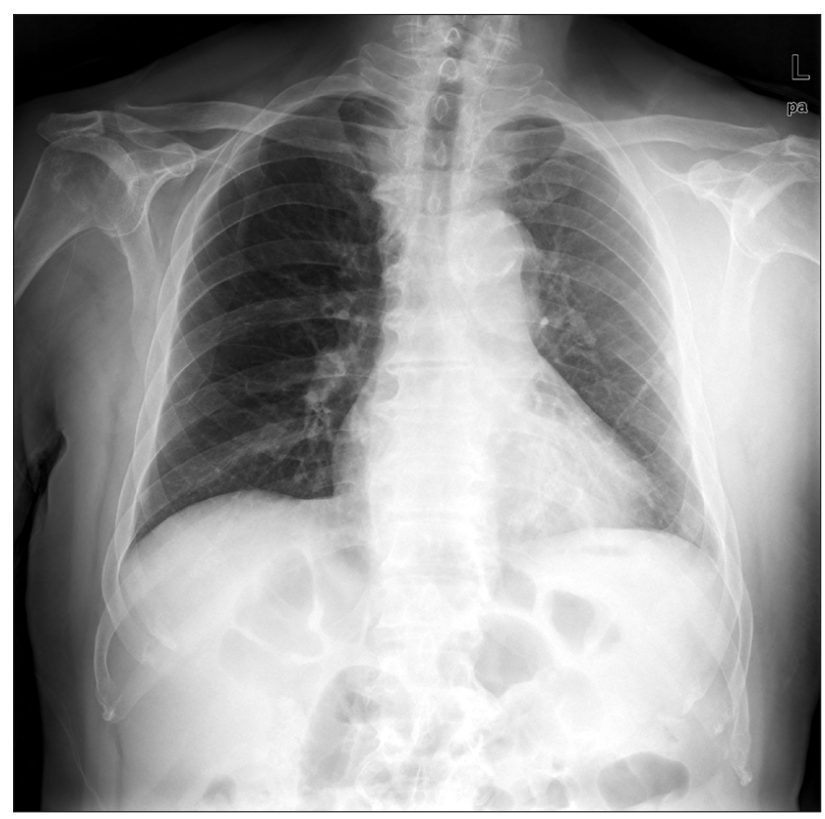

Fig. 2. Chest X-ray on POD 1 showing no remarkable findings except a little increased opacity on left chest wall. 
opacity in the left anterior chest wall (Fig. 2). In addition, there were no remarkable findings from the laboratory examinations. However, $10 \mathrm{mg}$ of furosemide was administered when the urine output decreased, even though the fluid intake was adequate.

On POD 2, the patient experienced syncope on his way to bathroom, possibly due to orthostatic hypotension. His vital signs were as follows: blood pressure (BP), 80/50 $\mathrm{mmHg}$; heart rate (HR), $142 \mathrm{bpm}$; and $\mathrm{SpO}_{2}, 85 \%$. Subsequently, the patient was transferred to the ICU and a diagnostic workup was performed. The ABGA revealed severe metabolic acidosis ( $\mathrm{pH}, 6.93$; $\mathrm{PaCO}_{2}, 28 \mathrm{mmHg} ; \mathrm{PaO}_{2}, 164 \mathrm{mmHg} ; \mathrm{HCO}_{3}, 5.9 \mathrm{mM}$; oxygen saturation, $98 \%$ ), and the patient received $15 \mathrm{~L} / \mathrm{min}$ oxygen via a reservoir mask. His $\mathrm{Hb}$ level decreased from $14.3 \mathrm{~g} / \mathrm{dl}$ to $9.6 \mathrm{~g} /$ dl. After transferring him to the ICU, his BP was $61 / 44 \mathrm{mmHg}$. Volume resuscitation was initiated with normal saline and packed red blood cells (RBCs). However, inotropes were not administered to the patient, because his BP increased to 90/50 $\mathrm{mmHg}$ after volume resuscitation. His hemodynamic values returned to normal with fluid therapy $(4,300 \mathrm{ml}$ of normal saline) and transfusion ( 3 units of packed RBC). In addition, sodium bicarbonate was administered to cure the severe acidosis. A computed tomography (CT) revealed a huge hematoma between the pectoralis major and minor muscles on the left chest wall (Fig. 3). However, an angiogram showed no detectable extravasations from the branches of the left subclavian artery. Laboratory tests revealed multiple organ dysfunction syndrome including respiratory failure, ischemic hepatitis, and acute kidney injury due to hemorrhagic shock. The levels of serum aspar-

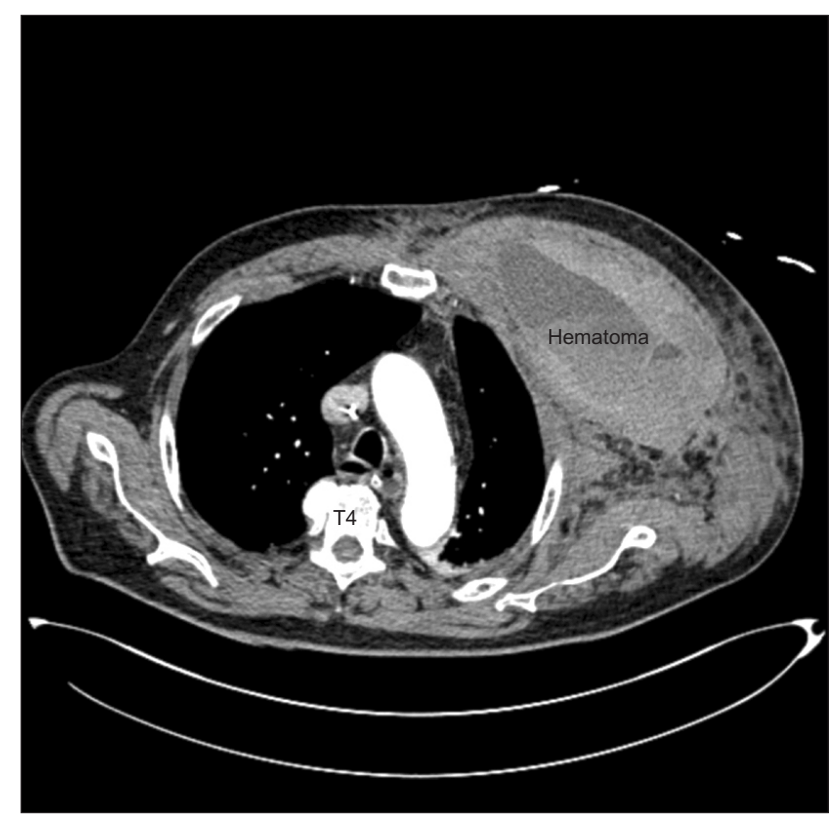

Fig. 3. Computed tomographic angiography of chest showing a huge hematoma on left anterior chest wall. tate aminotransferase (AST), alanine aminotransferase (ALT), lactate dehydrogenase (LDH), and creatinine $(\mathrm{Cr})$ were remarkably elevated: AST, 2,800 IU/L; ALT, 2,100 IU/L; LDH, 6,000 IU/L; $\mathrm{Cr}, 3.40 \mathrm{mg} / \mathrm{dl}$. Continuous renal replacement therapy (CRRT) was started on POD 2, and tracheal intubation was performed on POD 3 when an ineffective breathing pattern was observed and the $\mathrm{SpO}_{2}$ decreased to $90 \%$. Mechanical ventilation was applied without any intervention to the hematoma, such as insertion of the wound, use of a suction tube, or surgical removal. The patient was extubated on POD 12, and CRRT was stopped on POD 13. The patient was transferred to the general ward on POD 20, and the hospital course was uneventful thereafter.

\section{Discussion}

Central venous catheterization can be life saving, but can also cause complications such as pneumothorax, inadvertent puncture or perforation of great vessels, air embolism, catheter malposition, infection, and thrombus formation [1,2]. In this report, we describe a case of hemorrhagic shock caused by a huge hematoma after insertion of a CVC without apparent arterial puncture in a patient on anticoagulation therapy. Signs such as the decrease in urine output, orthostatic hypotension, compensatory increase of $\mathrm{HR}$, and aggravation of AF were associated with hypovolemia caused by hemorrhage, which was the cause of shock. However, we could not entirely rule out the possibility of obstructive shock due to the mass effect of the huge hematoma.

Complication rates during central venous catheterization have been significantly associated with the number of needle passes [2-4]. Approximately $40-50 \%$ of complication rates are associated with more than 2 punctures $[3,4]$. Successful catheterization at the first attempt limits the risk of immediate complications such as arterial puncture and pneumothorax, to less than $1 \%$ [4]. In our case, the insertion of a CVC was succeeded after a third needle passage. However, we did not note any signs associated with complications, such as pulsatile flow or air bubbles, during the procedure. Similarly, there were no abnormal findings such as extravasations of subclavian artery on the angiogram. Nonetheless, we could not completely rule out the possibility of arterial puncture around the subclavian region, because CT showed that the chest wall was severely compressed by the huge hematoma. Furthermore, small swelling and oozing were also observed at the insertion site after surgery. Therefore, the possibility of arterial puncture influenced the treatment decision for the hematoma in our case. However, we did not use a suction device or surgical removal for hematoma management because we assumed that an undetectable vessel injury might have caused the hematoma, leading to compression of the chest wall. In addition, we believed that respiratory failure might re- 
sult from multiorgan dysfunction syndrome caused by hemorrhagic shock, and not from compression of chest wall due to the huge hematoma.

Therefore, to reduce the complications associated with insertion of a CVC, ultrasound-guided central venous catheterization is strongly recommended. It could result in an initial high success rate with fewer needle passage attempts, faster access, and a reduction in mechanical complications $[5,6]$.

The choice of insertion site also depends on the incidence and type of complication. Internal jugular vein (IJV) and subclavian vein (SCV) catheterization have a similar overall risk of mechanical complications such as arterial puncture, hematoma, and pneumothorax [7]. However, SCV catheterization is more likely to be complicated by pneumothorax or hemothorax, whereas IJV catheterization is mostly associated with arterial puncture. In contrast, femoral vein (FV) catheterization has a higher incidence of mechanical complications than SCV or IJV [8]. Therefore, hematoma and arterial puncture are common during FV catheterization. The policy for central venous catheterization in our department advocates the SCV approach, because it is associated with a lower infection rate and better compliance than other approaches. However, it is difficult to apply pressure around the SCV as compared to the IJV or FV, which are easily compressible access sites that can be used when an arterial puncture or hematoma occurs. Therefore, the SCV catheterization technique should be particularly avoided, especially for patients on anticoagulation therapy or with coagulation disorders.

Arterial puncture, hematoma, and pneumothorax are the most common complications during central venous catheterization. The incidences of arterial puncture and hematoma are $3.1-4.9 \%$ and $1.2-2.1 \%$, respectively, in patients undergoing central venous catheterization [7]. This technique can lead to substantial morbidity, although these complications are usually self-limiting. Furthermore, other serious complications, including hemothorax requiring closed thoracostomy or hemorrhage requiring transfusion, show evident symptoms such as dyspnea and severe hypotension, and their progression is rapid; therefore, they are easily identified at the early stage. In our case, bleeding did not spread into the pleural space but between the pectoralis major and minor muscles in the left chest wall, and a huge hematoma was formed. In addition, the hematoma was too deep to cause an evident change in skin color or swelling, and the patient did not present any symptoms that could be considered serious complications. The abnormal finding of a small increased opacity in the left chest wall was dismissed as inconsequential, because the patient did not have any remarkable changes in hemodynamic values or laboratory findings. Thus, the hematoma was diagnosed only after hemorrhagic shock occurred. Anticoagulation therapy during the perioperative period might also have influenced the formation of a huge hematoma. Shinzato et al. [9] reported that hemorrhagic shock is associated with a huge hematoma after catheterization in patients with anticoagulant therapy. In our case, there was a minor association between warfarin therapy, which was administered preoperatively, and formation of the hematoma, as the value of PT (INR) was within the normal range. However, administration of heparin during the intraoperative and postoperative period might also be linked closely to the formation of a huge hematoma, especially if vessel injury occurs during insertion of CVC.

In conclusion, it is often difficult to detect serious complication such as concealed hematoma after central venous catheterization. Therefore, when insertion of a CVC is performed in patients requiring anticoagulant therapy, the possibility of massive bleeding must be considered and particular attention must be given to the care of such patients. Additionally, it might be helpful to prevent complications by choosing a proper site of insertion and ultrasound-guided technique.

\section{Acknowledgments}

This work was supported by the year 2012 clinical research grant from Pusan National University Hospital.

\section{References}

1. Taylor RW, Palagiri AV. Central venous catheterization. Crit Care Med 2007; 35: 1390-6.

2. Kusminsky RE. Complications of central venous catheterization. J Am Coll Surg 2007; 204: 681-96.

3. Eisen LA, Narasimhan M, Berger JS, Mayo PH, Rosen MJ, Schneider RF. Mechanical complications of central venous catheters. J Intensive Care Med 2006; 21: 40-6.

4. Lefrant JY, Muller L, De La Coussaye JE, Prudhomme M, Ripart J, Gouzes C, et al. Risk factors of failure and immediate complication of subclavian vein catheterization in critically ill patients. Intensive Care Med 2002; 28: 1036-41.

5. Fragou M, Gravvanis A, Dimitriou V, Papalois A, Kouraklis G, Karabinis A, et al. Real-time ultrasound-guided subclavian vein cannulation versus the landmark method in critical care patients: a prospective randomized study. Crit Care Med 2011; 39: 1607-12.

6. Karakitsos D, Labropoulos N, De Groot E, Patrianakos AP, Kouraklis G, Poularas J, et al. Real-time ultrasound-guided catheterisation of the internal jugular vein: a prospective comparison with the landmark technique in critical care patients. Crit Care 2006; 10 : R162. 
7. McGee DC, Gould MK. Preventing complications of central venous catheterization. N Engl J Med 2003; 348: 1123-33.

8. Merrer J, De Jonghe B, Golliot F, Lefrant JY, Raffy B, Barre E, et al. Complications of femoral and subclavian venous catheterization in critically ill patients: a randomized controlled trial. JAMA 2001; 286: 700-7.

9. Shinzato T, Fukui M, Kooguchi K, Sakaguchi M, Joo WJ. Hemorrhagic shock 3 days after catheterization from the axillary vein. J Anesth 2010; 24: 290-2. 